\title{
OPEN RETRACTIONS ON LOCALLY CONNECTED CONTINUA
}

\author{
S. MIKLOS
}

\begin{abstract}
It is shown that a locally connected continuum $X$ admits open retractions onto each of its subcontinua if and only if $X$ is either an arc or a simple closed curve.
\end{abstract}

We consider continua $X$ have the following property $\Gamma$ :

( $\Gamma$ ) $X$ admits open retractions onto each of its subcontinua.

Examples of continua having property $\Gamma$ are:

1. An arc and a simple closed curve.

2. A symmetrical topologist's sine curve $K$, i.e., $K=P \cup Q$, where $P$ is the closure of the set $\left\{(x, y) \in R^{2}: y=\sin 1 / x\right.$ and $\left.x \in(0,1 / \pi]\right\}$, and $Q$ is the image of $P$ under the symmetry with respect to the line $x=1 / \pi$.

3. The simplest indecomposable plane continuum (see [2, p. 204]).

4. A solenoid.

Problem. Characterize all continua having the property $\Gamma$.

In this paper we obtain a partial solution, for locally connected continua.

THEOREM. A locally connected continuum has the property $\Gamma$ if and only if it is either an arc or a simple closed curve.

We need the following lemmas.

LEMma 1. Let $X$ be a hereditarily locally connected continuum (i.e., every subcontinuum is locally connected) and let $Y$ be a subcontinuum of $X$ such that $X \backslash Y$ has infinitely many components. Then there exists a subcontinuum $Z$ of $X$ such that $Z \supsetneq Y$, $X \backslash Z$ has infinitely many components, and for every component $C$ of $X \backslash Z, Z \backslash \bar{C}$ is connected.

Proof. Let $K$ be a component of $X \backslash Y$, and consider the continuum $Y \cup K$. Choose $p \in K$, put $\varepsilon=d(p, Y)$, the distance from $p$ to $Y$, and let $\mathscr{U}$ be a finite open connected cover of the locally connected continuum $Y \cup K$ (in $Y \cup K$ ) with mesh $\mathscr{U}<\varepsilon / 2$. Let $\left\{U_{1}, U_{2}, \ldots, U_{n}\right\}$ be a subcollection of $\mathscr{U}$ covering $Y$ such that every $U_{i}$ intersects $Y$. Then the union $U_{K}=\bigcup\left\{U_{i}: i \in\{1,2, \ldots, n\}\right\}$ contains $Y$ and is connected. Thus the continuum $\bar{U}_{K}$ is such that $Y \subsetneq \bar{U}_{K} \subset(Y \cup K) \backslash\{p\}$ and,

Received by the editors April 11, 1983 and, in revised form, August 30, 1983.

1980 Mathematics Subject Classification. Primary 54C10, 54C15; Secondary 54F15, 54F25.

Key nords and phrases. Graph, $\Theta$-space, locally connected continuum, hereditarily locally connected continuum, open mapping, retraction.

1984 American Mathematical Society $0002-9939 / 84 \$ 1.00+\$ .25$ per page 
for each component $C$ of $(Y \cup K) \backslash \bar{U}_{K}$ we have $U_{K} \subset \bar{U}_{K} \backslash \bar{C} \subset \bar{U}_{K}$, whence we conclude $\bar{U}_{K} \backslash \bar{C}$ is connected.

Further, let $\left\{K_{t}\right\}_{t \in T}$ be the family of all components of $X \backslash Y$. For each index $t \in T$ we take this set $U_{K_{t}}$ defined as above, and we define $Z=\bigcup\left\{\bar{U}_{K_{t}}: t \in T\right\}$. Then $Z$ is obviously connected, and by local connectedness of $X$ it is a continuum. Hence we have $Y \subsetneq Z \subset X$ and $X \backslash Z$ has infinitely many components. Let $C$ be any of them. Then there is an index $s \in T$ such that $C$ is a component of $\left(Y \cup K_{s}\right) \backslash \bar{U}_{K_{s}}$ with $C \subset K_{s}$ and $\cup_{t \in T \backslash\{s\}} \bar{U}_{K_{t}} \cup U_{K_{s}} \subset Z \backslash \bar{C} \subset Z$. Thus $Z \backslash \bar{C}$ is connected, and the proof is complete.

LEMMA 2. Let $X$ be a locally connected continuum, $Y$ a proper subcontinuum of $X$, and $C$ a component of $X \backslash Y$ such that $Y \backslash \bar{C}$ is connected, and suppose there exists an open retraction $r: X \rightarrow Y$. Then $r(\bar{C})=Y$.

Proof. $r(C)$ is a nonempty open subset of $Y$, since $C$ is a nonempty open subset of $X$. Thus $r(C) \backslash \bar{C}$ is nonempty open in $Y \backslash \bar{C}$. We have $r(C) \backslash \bar{C}=r(\bar{C}) \backslash \bar{C}$, since $r(\bar{C})=(r(C) \backslash \bar{C}) \cup(Y \cap \bar{C})$. Thus $r(C) \backslash \bar{C}$ is also closed in $Y \backslash \bar{C}$. Since $Y \backslash \bar{C}$ is connected, we must have $r(C) \backslash \bar{C}=Y \backslash \bar{C}$. Hence

$$
r(\bar{C})=(r(C) \backslash \bar{C}) \cup(Y \cap \bar{C})=(Y \backslash \bar{C}) \cup(Y \cap \bar{C})=Y .
$$

A continuum is called a $\Theta$-space [1] if the complement of every subcontinuum has only finitely many components. It is known that a locally connected continuum is a $\Theta$-space if and only if it is a graph [1]. We use this to prove the following

Proposition 1. Every locally connected continuum with the property $\Gamma$ is a graph.

Proof. Let $X$ be any locally connected continuum having the property $\Gamma$. Suppose that $X$ is not a $\Theta$-space. Then there exists a subcontinuum $Y$ of $X$ such that the family of all components of $X \backslash Y$ is infinite. Since $X$ has the property $\Gamma$, it is hereditarily locally connected. Thus, by Lemma 1 , there exists a subcontinuum $Z$ of $X$ with $Y \subsetneq Z$ such that the family $\mathscr{C}$ of all components of $X \backslash Z$ is infinite, and such that for every member $C$ of $\mathscr{C}$ the set $Z \backslash \bar{C}$ is connected. Since $X$ has the property $\Gamma$, there exists an open retraction $r: X \rightarrow Z$ from $X$ onto $Z$. By Lemma 2 the closure of every member of $\mathscr{C}$ is mapped onto $Z$. By compactness of $X$, there exists a sequence $\left\{\bar{C}_{i}\right\}$ of closures of members of $\mathscr{C}$, with each $C_{i}$ contained in a distinct component of $X \backslash Y$, such that $\left\{\bar{C}_{i}\right\}$ converges to a compactum $D$. Since the components of $X \backslash Y$ are open sets we must have $D \subset Y$. Since $r\left(\bar{C}_{i}\right)=Z$ for each $i, r(D)=Z$. But $r(D)=D \subsetneq Z$, a contradiction. Thus $X$ is a $\Theta$-space and therefore a graph.

Proposition 2. Every graph with the property $\Gamma$ is either an arc or a simple closed curve.

Proof. Let $X$ be a graph with property $\Gamma$. We show that $X$ has no ramification points, and is therefore an arc or a simple closed curve. It is easily seen that $X$ contains an $\operatorname{arc} \alpha$, no point of which is a ramification point, such that $X \backslash \alpha$ is connected. Then $Y=X \backslash$ int $\alpha$ and $C=$ int $\alpha$ satisfy the hypotheses of Lemma 2, and every open retraction $r: X \rightarrow Y$ must take $\bar{C}=\alpha$ onto $Y$. Now suppose $X$ has a 
ramification point $y$. Then $y \in Y$, hence $y=r(p)$ for some $p \in \alpha$. Since $p$ is a nonramification point, there exists a sufficiently small closed neighborhood $V$ of $p$ in $X$ such that $V$ has at most two boundary points in $X$, while $r(V)$ is a closed neighborhood of $y$ with more than two boundary points in $Y$. Thus $r$ must take some interior point of $V$ onto a boundary point of $r(V)$, contradicting the openness of $r$.

The proof of the theorem follows from Example 1 and the above propositions.

The examples 1,2,3 and 4 suggest the following

Question. Is every continuum with the property $\Gamma$ atriodic?

Thanks are due to members of the topological seminar of Professor J. J. Charatonik for their criticism and many helpful remarks on the topic of this paper. The author is indebted also to Professor D. W. Curtis for making the paper short and more consistent.

\section{REFERENCES}

1. R. W. FitzGerald, Connected sets with a finite disconnection propert!, Studies in Topolngy (Proc. Conf. U.N.C., Charlotte, N.C., 1974), Academic Press, New York, 1975, pp. 139-173.

2. K. Kuratowski, Topologi, vol. II, Academic Press, New York, 1968.

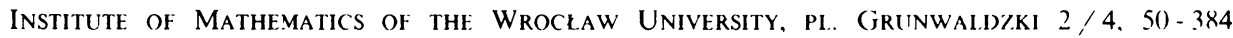
Wroclaw, POL.ANI) 